\title{
Post-operative Pain Outcomes of Laparoscopic Ventral Hernia Repair (LVHR): An eight-year experience
}

Shahid Rasul, Hassan Ahmed, Sanum Ali, Surrendar Dawani, Sarah Zahid, Sehrish Hussain, Salman Jafferi, Mansab Ali - - - - - - - - - - - - - - - - - - - - - - - - - - - - ABSTRACT:

Objective: To evaluate post-operative pain, recovery time and standard of living in patients undergoing LVHR in detail.

Study design and Setting: This prospective cohort study was conducted at a tertiary care hospital of Karachi, Pakistan, after getting approval from the "National Medical Centre Ethical Review Board", from January 2011 to December 2019,

Methodology: Total $n=577$ patients undergoing standard LVHR procedure (defect closed with non-absorbable monofilament suture, reinforced with intra-abdominal dual layer mesh, anchored with non-absorbable tacks \& sutures). Patient demographics, perioperative \& postoperative findings and post-operative pain analysis were investigated and presented as descriptive statistics. Follow-up was carried out at $1^{\text {st }}$ week, $2^{\text {nd }}$ week, $3^{\text {rd }}$ monthly, 6 monthly and 12 monthly post-operative appointments.

Results: During the study period of nine years (January 2011 to December 2019), 577 patients (primary ventral hernia $n=232$, recurrent ventral hernia $n=188$ patients, incisional hernia $n=157$ ) underwent LVHR. Mean post-operative hospital stay was $1.53 \pm 1.8$ days. Mean post-operative pain assessment on visual analog scale (VAS) after surgery (0-3days) was reported to be $38.5 \pm 29$ by 65 patients out of 577 (11.26\%), which significantly decreased at the end of 1 st week to $27.9 \pm$ 25.6. Only 3 patients $(0.51 \%)$ reported chronic pain during the span of 3-6 months.

Conclusion: LVHR was associated with considerably less post-operative pain, shorter hospital stay and reduced time of convalescence. It is demonstrated that LVHR to be a safe and superior approach for the repair ventral hernias.

Keywords: Chronic pain, Laparoscopic ventral hernia repair, Post-operative pain, Ventral hernia, Visual analog scale

- - - - - - - - - - - - - - - - - - - - - - - -

How to cite this Article:

Rasul S, Ahmed H, Ali S, Dawani S, Zahid S, Hussain S, Jaffri S, Ali M. Post-operative Pain Outcomes of Laparoscopic Ventral Hernia Repair (LVHR):An eight-year experience. J Bahria Uni Med Dental Coll.2021;11(4):148-152.DOI: https://doi.org/10.51985/JBUMDC2021048

This is an Open Access article distributed under the terms of the Creative Commons Attribution Non Commercial License (http:// creativecommons/org/licences/by-nc/4.0) which permits unrestricted non commercial use, distribution and reproduction in any medium, provided the original work is properly cited.

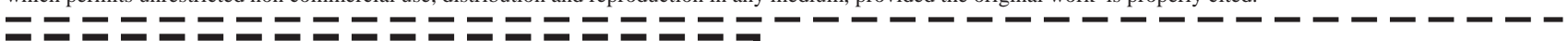

Shahid Rasul

I Professor Department of Surgery (Surgical Ward 21, Unit-4)

I Jinnah Postgraduate Medical Centre, Karachi

I Hassan Ahmed

Post-Fellowship Trainee (Surgical Ward 21, Unit-4)

Jinnah Postgraduate Medical Centre, Karachi

1

- Sanum Ali

- Assistant Professor, Department of Anatomy

I Basic Medical Sciences Institute (BMSI),

Jinnah Postgraduate Medical Centre, Karachi

Email: drdahri@gmail.com

Surrendar Dawani

Assistant Professor (Surgical Ward 21, Unit-4)

I Jinnah Postgraduate Medical Centre, Karachi

ISarah Zahid

I Post Graduate Trainee, Department of Anatomy

Basic Medical Sciences Institute (BMSI),

Jinnah Postgraduate Medical Centre, Karachi

I

- Sehrish Hussain

Post Graduate Trainee, Department of Anatomy

I Basic Medical Sciences Institute (BMSI),

I Jinnah Postgraduate Medical Centre, Karachi

I Salman Jafferi

Post-Fellowship Trainee (Surgical Ward 21, Unit-4)

Jinnah Postgraduate Medical Centre, Karachi

Mansab Ali

Senior registrar (Surgical Ward 21, Unit-4)

I Jinnah Postgraduate Medical Centre, Karachi

Received: 20-Aug-2021

Accepted: 28-Sep-2021

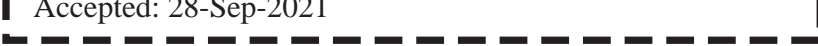

INTRODUCTION:

One of the most common pathologies presenting in the surgical clinic is Ventral hernia, appearing as a bulge through an opening in the anterior abdominal wall. ${ }^{1}$ Ventral hernia is a broad term and can be categorized as; epigastric, umbilical, paraumbilical, subcostal and Spigelian hernias and others, while incisional hernia is acquired in nature and occur at the site of any previous surgery through abdominal wall musculature. ${ }^{2}$

Ventral hernias are associated with abdominal discomfort, pain and deformed body image, leading to impaired patient's standard of living by creating hindrance in carrying out routine activities. ${ }^{3}$ More than 300,000 open ventral hernia repairs (OVHR) are performed in the United States each year for the repair of primary ventral hernia ${ }^{4}, 2-30 \%$ of them result in the development of incisional hernia, requiring approximately 90,000 repair procedures annually for its correction. ${ }^{5}$

Laparoscopic ventral hernia repair (LVHR) was introduced by LeBlanc and Booth in early 1990s and now it is being considered as a well-established procedure for the treatment of ventral hernia. ${ }^{6}$ The advantages it has over the conventional open repair include; minimal invasion, reduction in perioperative morbidity, less postoperative pain, reduced need of analgesics, shorter hospital stay and low recurrence 
rates followed by quick recovery. ${ }^{7}$ It also provides complete exploration of the abdominal cavity, making the parietal and visceral adhesiolysis easier, which is a basic step to maintain the stability of intestinal package and paramount factor in the reduction of the chronic abdominal pain, linked to the open repairs. ${ }^{8}$

In the recent years, attempts to evaluate the outcomes of different ventral hernia repair procedures have increased. LVHR has been extensively compared with OVHR for safety, morbidity and recurrence rates. ${ }^{9,10}$ However, there are only few studies in the literature evaluating incidence of acute $\&$ chronic pain and general well-being of patients post LVHR. This study was aimed to evaluate post-operative pain, recovery time and standard of living in patients undergoing LVHR in detail.

\section{METHODOLOGY:}

This prospective study was conducted among patients undergoing LVHR to characterize the repair of primary, recurrent and incisional ventral hernias, post-operative pain, period of recovery and standard of living in detail. Operative time, post-operative hospital stay, use of analgesics were also investigated for their potential association with postoperative pain.

After getting approval from the "National Medical Centre Ethical Review Board", this prospective cohort study was conducted from $1^{\text {st }}$ January 2011 to $31^{\text {st }}$ December 2019, at a tertiary care hospital in Karachi, Pakistan. Total 577 patients, ranging from 20 to 60 years of age were included and divided on the basis of etiology and type of hernia into three groups; Group A (Primary Ventral Hernia $n=232$ ), Group B (Recurrent Ventral Hernia $n=88$ ) and Group $C$ (Incisional Hernia $n=157$ ). Patients suffering from strangulated and obstructed hernias were not included.

After taking written and informed consent, all patients underwent detailed clinical history, examination, investigations. General anesthesia fitness evaluation was obtained and standard LVHR was performed on all patients. All surgeries were performed under general anesthesia with patients in a supine position, arms tucked at the sides, urinary and nasogastric catheters placed for decompression. Preoperative prophylactic antibiotics were administered. Pneumoperitoneum was established using Veress needle, followed by insertion of optical port for the exploration of abdominal cavity to visualize the location of hernia defect and distribution of adhesions. Additional two 5mm assisting trocars were inserted under direct vision for the lysis of adhesions and reduction of hernial sac (Figure: I). Defect size was measured and closed with non-absorbable monofilament suture. Appropriately sized intra-abdominal dual layer Mesh (ePTFE \& Polypropylene) placed with an overlap of approximately $3-4 \mathrm{~cm}$, in all directions. Points of reference were marked on the mesh and corresponding site on abdominal wall, to aid in orientation. Mesh was anchored with four non-absorbable monofilament transabdominal sutures and was stapled with non-absorbable spiral tacks measuring $5 \mathrm{~mm}$. Ports were removed and skin incisions were closed. Postoperative analgesic protocol included Ketorolac (30mg) and Paracetamol (1g) I/V 8 hourly. Followup was carried out at $1^{\text {st }}$ week, $2^{\text {nd }}$ week, $3^{\text {rd }}$ monthly, 6 monthly and 12 monthly post-operative appointments.

\section{RESULTS:}

During the study period of nine months, 577 patients underwent LVHR. A total of 232 patients (40.2\%) for repair of primary ventral hernia, 188 repair of recurrent ventral hernia $(15.25 \%)$ and 157 incisional hernia $(27.20 \%)$. The mean age of patients in all groups was $42.07 \pm 17.93$ years and majority of patients were of female gender (55\%), as shown in Table- I. Mean post-operative hospital stay was found to be $1.53 \pm 1.8$ days. Parameters like operating time, estimated blood loss, analgesia requirement, return to daily activities \& work along with complications and recurrence rate can be observed in Table-1. Visual analog scale (VAS) was used on a $100 \mathrm{~mm}$ line, for the assessment of postoperative pain in patients after LVHR. Out of 577, 65 patients $(11.26 \%)$ complained of pain having mean VAS score of 38.5 \pm 29.3 , immediately after the surgery (0-3days). They were managed conservatively by I/M and oral doses of Ketorolac or NSAIDs. Out of those 65 patients, 20 (3.46\%) reported pain with mean VAS score of $27.9 \pm 25.6$ by the end of $1^{\text {st }}$ week, depicting a significant reduction in pain. Only 3 out of 65 patients $(0.51 \%)$ reported chronic pain during the span of 3-6 months (Table: II). They were prescribed oral doses of Ketorolac or NSAIDs.

\section{DISCUSSION:}

In the past, surgeons tended to focus on the outcomes of LVHR in terms of recurrence and complications. From a patient's perspective, however, pain and discomfort from the abdominal wall may be more important than the risk for recurrence. The ultimate surgical goal should be to restore or increase the standard of living by limiting the incidence

Figure. 1: Schematic representation of OVHR incision (A) and LVHR ports (B)

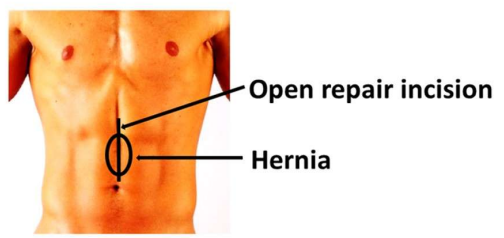

A: Open repair incision

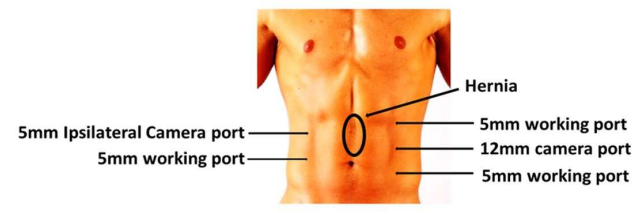

B:Laparoscopic Repair Port Placement 
Shahid Rasul, Hassan Ahmed, Sanum Ali, Surrendar Dawani, Sarah Zahid, Sehrish Hussain, Salman Jafferi, Mansab Ali

Table 1: Patient Demographics

\begin{tabular}{|c|c|c|c|c|}
\hline Variables & Overall & $\begin{array}{c}\text { Primary } \\
\text { ventral hernia }\end{array}$ & $\begin{array}{c}\text { Recurrent } \\
\text { ventral hernia }\end{array}$ & $\begin{array}{c}\text { Incisional } \\
\text { hernia }\end{array}$ \\
\hline Total no: of patients $(\%)$ & $577(100)$ & $232(40.2)$ & $88(15.25)$ & $157(27.20)$ \\
\hline Females (\%) & $317(55)$ & $162(69.82)$ & $51(57.95)$ & $119(75.79)$ \\
\hline Males $(\%)$ & $260(45)$ & $70(30.17)$ & $37(42.04)$ & $38(24.20)$ \\
\hline Age (mean \pm std $)$ & $42.07 \pm 17.93$ & $46.77 \pm 13.23$ & $45.82 \pm 14.18$ & $49.46 \pm 10.54$ \\
\hline BMI $($ mean \pm std $)$ & $29 \pm 6.4$ & $28 \pm 6.2$ & $31.92 \pm 6.10$ & $31.06 \pm 6.53$ \\
\hline \multicolumn{5}{|l|}{ PERIOPERATIVE FINDINGS } \\
\hline Defect width $\left(\mathrm{cm}^{2}\right)$ & $8(3-15)$ & $4.67(3-8)$ & $5(4-8)$ & $7.5(5-15)$ \\
\hline Operative time (min) & $100(80-120)$ & $87(75-100)$ & $100(80-120)$ & $110(90-130)$ \\
\hline Estimated blood loss (ml) & $30(10-50)$ & $25(10-40)$ & $28(10-40)$ & $34(10-60)$ \\
\hline Mesh size $\left(\mathrm{cm}^{2}\right)$ & $25 \times 20$ & $15 \times 10$ & $20 \times 15$ & $25 \times 20$ \\
\hline \multicolumn{5}{|l|}{ POST-OPERATIVE OUTCOMES } \\
\hline Post-operative hospital stay (days) & $1.53 \pm 1.8$ & $1.49 \pm 1.4$ & $1.32 \pm 1.5$ & $2.1 \pm 1.2$ \\
\hline Use of analgesics (doses) & $4(2-6)$ & $3(2-4)$ & $3(2-4)$ & $3(2-5)$ \\
\hline Return to daily activities (days) & $3.61(2-5)$ & $2(1-2)$ & $2(1-2)$ & $3(2-4)$ \\
\hline Return to work (days) & $7.13(6-10)$ & $5(5-7)$ & $5(5-7)$ & $6.5(6-10)$ \\
\hline Post-operative complications & $15(2.59 \%)$ & $2(0.34 \%)$ & $4(0.68 \%)$ & $9(1.53 \%)$ \\
\hline Recurrence & $7(1.21 \%)$ & - & $2(0.34 \%)$ & $5(0.86 \%)$ \\
\hline
\end{tabular}

BMI: Body mass index, std: Standard deviation

Table 2: Post-Operative Pain Analysis on Visual scale (VAS)

\begin{tabular}{|l|c|c|c|c|c|c|}
\hline Post-operative pain analysis & $\mathbf{0 - 3}$ days & At $\mathbf{1}$ week & At $\mathbf{2}$ weeks & 3 months & 6 months & 12 months \\
\hline Mean VAS score & $38.5 \pm 29.3$ & $27.9 \pm 25.6$ & $22.4 \pm 24.3$ & $8.8 \pm 15.3$ & $4.1 \pm 6.4$ & $2.7 \pm 4.2$ \\
\hline No of patients $(\boldsymbol{n}=\mathbf{5 7 7})$ & $65(11.26 \%)$ & $20(3.46 \%)$ & $08(1.38 \%)$ & $03(0.51 \%)$ & $01(0.17 \%)$ & $00(0 \%)$ \\
\hline
\end{tabular}

Interpretation of pain on 100mm Visual analogue scale (VAS): no pain (0-4), mild pain (5-44), moderate pain (45-74) and severe pain (75-100).

of post-operative pain and complications. Chronic abdominal pain (persisting for more than 3-4 months) ${ }^{11}$ is a serious problem after ventral hernia repair. Nerve injury or entrapment due to extensive adhesiolysis, dissection of the abdominal wall for myofascial structures post OVHR could lead to increased incidence of chronic pain. ${ }^{12}$

With the advent of minimally invasive procedures, such complications can be reduced. The present prospective study demonstrated that LVHR had a significant positive influence on a broad spectrum of recovery parameters. Even though large incision is not used, LVHR may be associated with significant abdominal pain. The incidence of chronic pain after LVHR has been reported to be approximately 1-3\% in literature, affecting the quality of life badly. ${ }^{13}$

Gronnier et al reported that after a mean follow-up period of $24.6 \pm 9.9$ months, 31 patients (28.4\%) complained of chronic pain post OVHR, which was predominantly neuropathic in nature. ${ }^{14}$ Eker et al found association of greater number of patients with post-operative in OVHR group than LVHR group ${ }^{15}$, however, our study witnessed an event free recovery by most of patients (table- II). During the span of 72 hours after LVHR, $11.26 \%$ of patients experienced post-operative pain which resolved in most of the patients by the end of 2 weeks. Only three patients $(0.51 \%)$ complained of chronic pain during $3^{\text {rd }}$ monthly appointment, no neuropathic involvement was seen and relief was attained by the administration of opioid analgesics, by the end of $6^{\text {th }}$ month.

Post-operative chronic pain is largely related to fixation of mesh with tacks or sutures. Pain due to fixation is different from that at the port sites. The postoperative pain produced by the fixation techniques could play an important role in deciding between sutures and tacks for mesh fixation. ${ }^{16} \mathrm{~A}$ randomized control trial from Sweden reported persistent post-operative pain in $7.4 \%$ of patients with only tacker fixation of mesh. ${ }^{17}$ In this study, both tackers and sutures for the fixation of mesh were used and found no incidence of chronic pain with tacker fixation. The observations regarding association of chronic pain with tacker fixation are in alliance with Liot $\mathrm{E}$, and her team who reported no change in the occurrence of chronic pain in group of patients with absorbable tackers, in comparison to non-absorbable 
tackers' group. ${ }^{18}$

Operating time did not differ significantly between LVHR \& OVHR as reported by Thota A, and his team that, laparoscopic repair took at an average of 94.35 minutes, while open mesh repair took 92.65 minutes. ${ }^{19}$ The mean duration of surgery in the study subjects undergoing LVHR was $100 \pm 20$ minutes, however, it slightly increased in cases undergoing LVHR for incisional hernia (110 \pm 20 minutes $)$. An Egyptian prospective study reported shorter hospital stay in patients that underwent LVHR $(1.94 \pm 0.67 \text { days })^{20}$, this was in agreement with our study in which patients were discharged from hospital after $1.53 \pm 1.8$ days. This could be considered as a valuable outcome owing to the fact that shorter hospital stays are associated with reduced hospital expenditure.

This study witnessed few postoperative complications $(2.59 \%)$, including seromas which were conservatively managed with antibiotics and no need for drain was required. No intestinal injury or obstruction was observed and no mortality took place, indicating that LVHR is a safe surgical procedure for primary and incisional ventral hernia repair. Moreover, no patients required reoperation for a port site hernia between the time of initial laparoscopic ventral hernia repair and assessment for the study.

An overall large number of patients, effective long-term follow-up, and the specific standardized operative methods are the main strengths of this study; however, our study lacks a comparison to an open surgery group. A substantial portion of patients were referred from other private set-ups and surgeons, which proves that minimally invasive surgical outcomes are much better then open repair.

\section{CONCLUSION:}

It is demonstrated from the experience that LVHR to be a safe and superior approach for the repair ventral hernias. It is better in terms of postoperative pain related complications and return to routine activities and work, yielding a good standard of living and patient's satisfaction, post-operatively. Although these results are encouraging, larger, long-term, multicenter studies comparing LVHR and open repair are needed.

Authors Contribution:
Shahid Rasul: Drafting of the work and Final approval of the |
version to be published
Hassan Ahmed: Drafting of the work and Final approval of
the version to be published
I Sanum Ali: conception or design of the work; or the acquisition, I
analysis, or interpretation of data for the work and Final approval
of the version to be published
I Surrendar Dawani: Acquisition \& analysis of data and Final I
approval of the version to be published
I Sarah Zahid: Acquisition \& analysis of data and Final approval
of the version to be published
Sehrish Hussain: Interpretation of data and Final approval of
the version to be published
I Salman Jafferi: Interpretation of data and Final approval of I
the version to be published
Mansab Ali: Drafting of the work and Final approval of the I
I version to be published

\section{REFERENCES:}

1. Mohamed Yousef A, Mohamed MA, Gaber A, Ahmed MS, Husein HM. Laparoscopic Ventral Hernia Repair: Tow Centre Experience Prospective Comparative Study. OAlib 2018; 5(09):1. DOI: https://doi.org/10.4236/oalib.1104871

2. Williams N, O'Connell PR, McCaskie AW editors. Bailey \& Love's Short Practice of Surgery. $27^{\text {th }}$ ed. CRC press; 2018. p.1036-1039

3. Colavita PD, Tsirline VB, Belyansky I, Walters AL, Lincourt $\mathrm{AE}$, Sing RF, et al. Prospective, long-term comparison of quality of life in laparoscopic versus open ventral hernia repair. Ann Surg 2012; 256(5):714-23. DOI: https://doi.org/ SLA.0b013e3182734130.

4. Kroese LF, Gillion JF, Jeekel J, Lange JF, Kleinrensink GJ, Members HC. Identification of risk factors for 30-day postoperative complications in patients undergoing primary ventral hernia repair: a prospective cohort study of 2,374 patients. Surgery. 2018; 163(5):1160-4.

5. Kumar D, Khan H, Qureshi MS. Outcome of four years experience in laparoscopic ventral hernia repair. Pak J Med Sci 2015; 31(4):987.

6. Mercoli H, Tzedakis S, D’Urso A, Nedelcu M, Memeo R, Meyer N, et al. Postoperative complications as an independent risk factor for recurrence after laparoscopic ventral hernia repair: a prospective study of 417 patients with long-term follow-up. Surg Endosc. 2017; 31(3):1469-77. DOI: https://doi.org/10.1007/s00464-016-5140-2

7. He C, Lu J, Ong MW, Lee DJ, Tan KY, Chia CL. Seroma prevention strategies in laparoscopic ventral hernia repair: a systematic review. Hernia 2020; 24(4):717-31.

8. Bernardi K, Olavarria OA, Holihan JL, Kao LS, Ko TC, Roth JS, et al. Primary fascial closure during laparoscopic ventral hernia repair improves patient quality of life: a multicenter, blinded randomized controlled trial. Ann Surg 2020; 271(3):434-9. DOI: https://doi.org/ 10.1097/SLA. 0000000000003505

9. Froylich D, Segal M, Weinstein A, Hatib K, Shiloni E, Hazzan D. Laparoscopic versus open ventral hernia repair in obese patients: a long-term follow-up. Surg Endosc. 2016; 30(2):6705 .

10. Awaiz A, Rahman F, Hossain MB, Yunus RM, Khan S, Memon B, Memon MA. Meta-analysis and systematic review of laparoscopic versus open mesh repair for elective incisional hernia. Hernia 2015; 19(3):449-63.

11. Ahmed MA, Tawfic QA, Schlachta CM, Alkhamesi NA. Pain and surgical outcomes reporting after laparoscopic ventral hernia repair in relation to mesh fixation technique: a systematic review and meta-analysis of randomized clinical trials. J Laparoendosc Adv Surg Tech A 2018; 28(11):1298-315.

12. Warren JA, McGrath SP, Hale AL, Ewing JA, Carbonell AM, Cobb IV WS. Patterns of recurrence and mechanisms of failure after open ventral hernia repair with mesh. Am Surg 2017; 83(11):1275-82.

13. Uranues S, Salehi B, Bergamaschi R. Adverse events, quality of life, and recurrence rates after laparoscopic adhesiolysis and recurrent incisional hernia mesh repair in patients with previous failed repairs. J Am Coll Surg 2008; 207(5):663-9.

14. Gronnier C, Wattier JM, Favre H, Piessen G, Mariette C. Risk factors for chronic pain after open ventral hernia repair by underlay mesh placement. World J Surg 2012; 36(7):154854. 
Shahid Rasul, Hassan Ahmed, Sanum Ali, Surrendar Dawani, Sarah Zahid, Sehrish Hussain, Salman Jafferi, Mansab Ali

15. Eker HH, Hansson BM, Buunen M, Janssen IM, Pierik RE, Hop WC, et al. Laparoscopic vs open incisional hernia repair: a randomized clinical trial. JAMA Surg 2013; 148(3):25963. doi:10.1001/jamasurg.2013.1466

16. Harsløf S, Krum-Møller P, Sommer T, Zinther N, Wara P, Friis-Andersen $\mathrm{H}$. Effect of fixation devices on postoperative pain after laparoscopic ventral hernia repair: a randomized clinical trial of permanent tacks, absorbable tacks, and synthetic glue. Langenbecks Arch Surg. 2018; 403(4):529-37.

17. Rogmark P, Petersson U, Bringman S, Ezra E, Österberg J, Montgomery A. Quality of life and surgical outcome 1 year after open and laparoscopic incisional hernia repair. Ann Surg. 2016; 263(2):244-50.
18. Liot E, Bréguet R, Piguet V, Ris F, Volonté F, Morel P. Evaluation of port site hernias, chronic pain and recurrence rates after laparoscopic ventral hernia repair: a monocentric long-term study. Hernia 2017; 21(6):917-23.

19. Thota A, Ravishankar KS, Rao KV, Ramesh BS, Ananda BB. Comparative study between open and laparoscopic ventral hernia repair: A prospective non-randomized single institutional study. J Dr NTR Univ Health Sci 2017; 6(4):217. DOI: https://doi.org/10.4103/JDRNTRUHS.JDRNTRUHS_115_16

20. Baghdady AS, Mostafa HA, Salem AE, Abdullah HA, Saad MR. Short Outcome Evaluation of Laparoscopic Ventral Hernia Repair Ahmed Saleh Mostafa Baghdady, Hussein Ali Mostafa. Egypt J Hosp Med. 2021; 83(1):1338-4. 